\title{
GLRT Based Anomaly Detection for Sensor Network Monitoring
}

\author{
Fouzi Harrou, Member, IEEE, Ying Sun \\ CEMSE Division, King Abdullah University of Science and Technology, Saudi Arabia \\ fouzi.harrou@kaust.edu.sa
}

\begin{abstract}
Proper operation of antenna arrays requires continuously monitoring their performances. When a fault occurs in an antenna array, the radiation pattern changes and can significantly deviate from the desired design performance specifications. In this paper, the problem of fault detection in linear antenna arrays is addressed within a statistical framework. Specifically, a statistical fault detection method based on the generalized likelihood ratio (GLR) principle is utilized for detecting potential faults in linear antenna arrays. The proposed method relies on detecting deviations in the radiation pattern of the monitored array with respect to a reference (fault-free) one. To assess the abilities of the GLR based fault detection method, three case studies involving different types of faults have been performed. The simulation results clearly illustrate the effectiveness of the GLRbased fault detection method in monitoring the performance of linear antenna arrays.
\end{abstract}

\section{INTRODUCTION}

The recent developments in wireless communication systems have greatly increased the demand for antenna arrays. The occurrence of faults in one or more elements in an antenna array changes the radiation pattern of the array, which degrades the performance of the entire array [1]. Thus, monitoring of antennas is essential for their proper and safe operation. Specifically, faults in antenna array need to be detected, isolated, and then removed before they affect the performance of the wireless system [2]. Two types of faults are usually encountered in antenna arrays: complete (or onoff ) faults and partial fault [3], [4]. Antenna elements with on-off faults do not radiate. This type of faults is widely encountered in practice. On the other hand, partial faults (which can be due to interferences and other factors) cause degradation in the desired radiation pattern. Generally, faulty array elements not only can distort the directivity of the antenna pattern, but also the side lobes levels of the radiation pattern [5], [6]. As a result, in the presence of fault(s), the radiation pattern can significantly deviate from the desired pattern indicating the presence of a new condition that is significantly distinguishable from the desired faultless working mode. Therefore, implementing fault detection techniques in antenna arrays is becoming more and more important [6], [7].

This paper presents a statistical method for detecting the presence of faults in a linear antenna array. This method is based on the principle of the generalized likelihood ratio (GLR) and is intended to reveal any drifts from the normal behavior of the monitored antenna array. The remainder of the

978-1-4799-7560-0/15/\$31 (c)2015 IEEE paper is organized as follows. Section II outlines some basic antenna array theory as it applies to this problem. Then, the GLR test which is commonly used in composite hypothesis testing is described in Section III. Then, a methodology for fault detection in antenna arrays is described in Section IV, followed by simulated examples that illustrate the performance of the GLR hypothesis testing method are presented in Section V. Finally, concluding remarks are presented in Section VI.

\section{LINEAR ARRAY ANTENNA}

Let us consider a 'fault-free' linear antenna array having an even number $(2 \mathrm{~N})$ of isotropic elements. Assuming that the elements are symmetrically placed and conjugate-symmetrically excited about the center of the array, the normalized radiation pattern of this array can be calculated using the following expression [8]:

$$
F_{s}(\varphi)=\frac{f(\varphi)}{F_{s \max }} \sum_{i=1}^{N} a_{i} \cos \left(k x_{i} \sin (\varphi)+\psi_{i}\right),
$$

where $f(\varphi)$ is the element pattern, $\varphi$ is the angular direction; $a_{i}$ and $\psi_{i}$ are the current excitation amplitude and phase of the $i^{t h}$ array element located at the position given by $x_{i}, k=\frac{2 \pi}{\lambda}$ is the wave number with a wavelength of $\lambda$, and the position $x_{i}$ can be computed using the inter-element spacing as follows: $x_{i}=\left(i-\frac{1}{2}\right) \Delta x, \quad i=1, N$.

Failure(s) in antenna arrays can severely distort the actual radiation pattern. As discussed earlier, two different kinds of faults can be distinguished: on-off and partial faults. In arrays with on-ff faults, the faulty elements do not radiate at all (i.e., fail completely), which is equivalent to assuming that their relative excitations are zero. However, in array with partial failures, the faulty elements don't completely fail completely, but radiate a fraction of its normal power. Partial faults my be caused by noise, interferences, or other factors. As more elements fail in the array, the pattern gets degraded further. Thus, detecting the presence of faults in antenna arrays is necessary to ensure their normal operation. A description of the GLR test, which is used to achieve this objective, is presented next.

\section{GENERALIZED LIKELIHOOD RATIO TEST}

The GLR test is an important statistical method that can be used to solve composite hypotheses testing problems by maximizing the likelihood ratio function over all possible faults [9], 
[10], [11]. The GLR test is based on the Neyman-Pearson theorem, which allows the maximization of the probability of detection for a given value of the false alarm probability [10]. In binary hypothesis testing, when hypotheses are composite or the corresponding data probability density functions (pdfs) contain unknown parameters, the generalized likelihood ratio (GLRT) test constitutes a popular means for deciding between the two possibilities.

Consider the fault detection problem, where a measured vector $Y \in \mathbb{R}^{n}$ follows one of the two Gaussian distributions: $\mathscr{N}\left(0, \sigma^{2} I_{n}\right)$ or $\mathscr{N}\left(\theta \neq 0, \sigma^{2} I_{n}\right)$, where $\theta$ is the mean vector (which is the value of the fault) and $\sigma^{2}>0$ is the variance, which is assumed to have a known value. The hypothesis testing problem seeks to decide between two hypotheses, the null hypothesis $\mathscr{H}_{0}$ and the alternative hypothesis $\mathscr{H}_{1}$ [12]:

$$
\begin{cases}\mathscr{H}_{0}=\left\{Y \sim \mathscr{N}\left(0, \sigma^{2} I_{n}\right)\right\} & \text { (null hypothesis); } \\ \mathscr{H}_{1}=\left\{Y \sim \mathscr{N}\left(\theta, \sigma^{2} I_{n}\right)\right\}, & \text { (alternative hypothesis). }\end{cases}
$$

The GLR test is based on the evaluation of the generalized likelihood ratio of $Y, \Lambda(Y)$, which is defined as the ratio between the probability density function (pdf) of $Y$ under $\mathscr{H}_{1}$, and the pdf of $Y$ under $\mathscr{H}_{0}$. The GLR test estimates the unknown parameter, $\theta$, using maximum likelihood estimation by maximizing the generalized likelihood ratio $\Lambda(Y)$ as follows [12]:

$$
\begin{aligned}
\Lambda(Y) & =2 \log \frac{\sup _{\theta \in \mathbb{R}^{n}} f_{\theta}(Y)}{f_{\theta=0}(Y)} \\
& =\frac{1}{\sigma^{2}}\left\{\|Y-\widehat{\theta}\|_{2}^{2}+\|Y\|_{2}^{2}\right\} \\
& =\frac{1}{\sigma^{2}}\left\{\|Y\|_{2}^{2}\right\}
\end{aligned}
$$

where $\widehat{\theta}=\arg \min _{\theta}\|Y-\theta\|_{2}^{2}=Y$ is the maximum likelihood estimate of $\theta,\|.\|_{2}$ represents the Euclidean norm, and $f_{\theta}(Y)=\frac{1}{(2 \pi)^{\frac{n}{2}} \sigma^{n}} \exp \left\{-\frac{1}{2 \sigma^{2}}\|Y-\theta\|_{2}^{2}\right\}$ is the probability density function of $Y$. It is important to note that in the above derivation, we maximized the likelihood function by maximizing its natural logarithm due to the fact that the logarithmic function is monotonic [12]. The GLR test makes this decision between the null or alternative hypotheses by comparing between $\Lambda(Y)$ and a given value of the threshold $h(\alpha)$.

$$
\Lambda(Y)=\frac{1}{\sigma^{2}}\left\{\|Y\|_{2}^{2}\right\} \underset{\mathscr{H}_{0}}{\mathscr{H}_{1}} h(\alpha) .
$$

If $\Lambda(Y)$ is larger than $h(\alpha)$, the GLR test decides for $\mathscr{H}_{1}$, otherwise $\mathscr{H}_{0}$ is assumed to be true. This means that knowing the distribution of the decision function $\Lambda(Y)$ under the null hypothesis $\mathscr{H}_{0}$ allows the design of a statistical test with a desired false alarm rate, $\alpha$, where the threshold $h(\alpha)$ is selected to satisfy the following false alarm probability [12]:

$$
\mathbb{P}_{0}(\Lambda(Y) \geq h(\alpha))=\alpha
$$

where, $\mathbb{P}_{0}(A)$ is the probability of an event $A$ when $Y$ is distributed according to the null hypothesis $\mathscr{H}_{0}$. Since $Y$ is assumed to be normally distributed (see equation 2 ), the statistic $\Lambda$ follows a $\chi^{2}$ distribution with $(n-1)$ degrees of freedom. This chi-square distribution is central under $\mathscr{H}_{0}$ and noncentral under $\mathscr{H}_{1}$ with a parameter of non-centrality equal to: $\kappa_{\theta}=\frac{1}{\sigma^{2}}\|\theta\|_{2}^{2}$. Also, the power function of $\delta$ can be computed as follows [12]:

$$
\beta_{\delta}=\mathbb{P}_{\theta}\left(\delta(Y)=\mathscr{H}_{1}\right) .
$$

Next, the GLR test described earlier will be used to detect faults in linear antenna arrays.

\section{FAULT DETECTION IN LINEAR ANTENNA ARRAY USING GLR TEST}

In this section, the GLR test will be used to detect faults in radiation pattern of linear antenna arrays. Once obtained, a reference radiation pattern of the fault-free antenna array can be used to detect unusual conditions, such as on-off and partial faults. The differences or residuals between the reference pattern and the actual pattern of the monitored array normally has zero mean and a variance related the amount of noise measurements. When a radiation pattern is faulty, the mean of its associated residuals deviates from its nominal value, which can be detected using GLR hypothesis testing. Fault detection in linear antenna array using GLR hypothesis testing involves the following steps: 1) the generation of residuals, 2) the evaluation of these residuals using a GLR test.

Let $\mathbf{e}=\left[e_{\varphi_{1}}, \ldots, e_{\varphi_{i}}, \ldots, e_{\varphi_{n}}\right]$ be the residual vector, which is defined as the difference between the reference radiation pattern and the radiation pattern of the tested or monitored array. In the absence of a fault, the residual vector equals,

$$
\mathbf{e}=F_{d}(\varphi)-F_{s}(\varphi)
$$

and in the presence of an additive fault vector, $\theta$, the residual vector equals,

$$
\mathbf{e}=F_{d}(\varphi)-F_{s}(\varphi)[+\theta] .
$$

The residual vector defined in equation (6) is assumed to follow a Gaussian distribution. Thus, the fault detection problem can be thought of as detecting the presence of an additive bias vector, $\theta$, in the residual vector, $\mathbf{e}$, which can be treated as a hypothesis testing problem, considering two hypotheses: the null hypothesis $\mathscr{H}_{0}$, where $\mathbf{e}$ is fault-free and the alternative hypothesis $\mathscr{H}_{1}$, where e contains a fault. The formulation of this hypothesis testing problem can be written as follows:

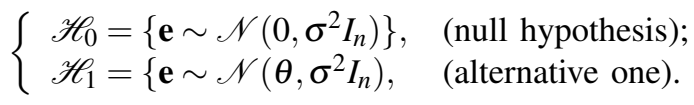

An algorithm that outlines the proposed GLR fault detection method in linear antenna arrays is summarized in Table I. 
TABLE I

ANTENNA ARRAY MONITORING USING A GLR FAULT DETECTION ALGORITHM.

\begin{tabular}{cl}
\hline Step & Action \\
\hline 1) & Given: \\
& Training fault-free radiation pattern data (obtained from \\
& a normally operating antenna array), and testing radiation \\
& pattern data (obtained from an array possibly containing \\
& faulty elements), \\
& A fixed false alarm probability, $\alpha$, \\
2) & Synthesize the radiation pattern with the desired perfor- \\
& mance, \\
3) & Compute the residual vectors, $\mathbf{e}$, \\
4) & Compute the decision function $\Lambda(\mathbf{e})$, \\
5) & Compute the decision threshold $h(\alpha)$ for the GLR test, \\
6) & Check for faults: if $\Lambda(\mathbf{e}) \geq h(\alpha)$, then declare anomaly. \\
&
\end{tabular}

\section{Simulated Example}

In this section, the performance of the GLR fault detection algorithm will be assessed through three different cases studies representing different faults. In the first case study, it is assumed that the monitored linear array contains two completely failing elements (case A). In the second case study, an antenna array with partial failures is considered (case B). In third case study, the radiation pattern of the monitored linear array is corrupted by additive random Gaussian noise (case C).

The fault-free pattern for this example is generated by simulating the radiation pattern of a uniformly spaced linear array constituted with 12 rectangular microstrip antennas. The radiation pattern $f(\varphi)$ used in this simulations is the same as the one described in [13], [14], [4]. This radiation pattern is determined for a substrate with a permittivity of 2.2 , a thickness of $0.152 \mathrm{~cm}$, a square patch with a dimension of $1.89 \mathrm{~cm}$, and a frequency of $5 \mathrm{GHz}$.

1) Case A: detection of complete failures: This first case study is aimed to assess the ability of the GLR test to detect the presence of on-off faults in the linear antenna array. We consider that the array defective elements fail completely, which is equivalent to assuming that their relative excitations are zero. Two examples are presented here to illustrate the ability of the GLR fault detection method to detect complete faults in linear antenna arrays. In the first example, we have considered a linear array with two completely failing element at positions ( -3 and 3 ) as shown in Figure 1. The radiation pattern is represented using 180 samples between angles of 90 and 90 degrees. To better represent randomness in practical measurements, zero-mean Gaussian noise with a standard deviations of $\sigma=0.005$ is added to the simulated radiation pattern. Figure 2 shows the reference radiation pattern of fault-free antenna array obtained using the Minimax algorithm (solid curve) and the faulty pattern (circle-dash curve). One can clearly observe from Figure 2, that due to the failing element, the radiation pattern deviated from the reference pattern.

The GLR threshold value is found to be $h(\alpha)=0.004$ for a false alarm probability of $\alpha=5 \%$. The GLR decision function

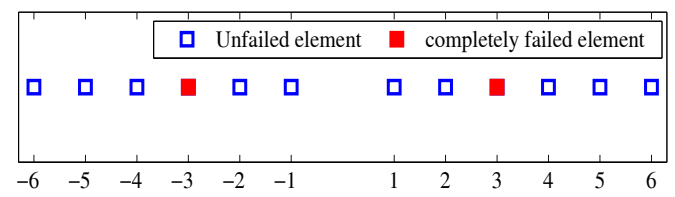

Fig. 1. Example of a linear antenna array with two completely failing elements at positions -3 and 3 (Case A, first example).

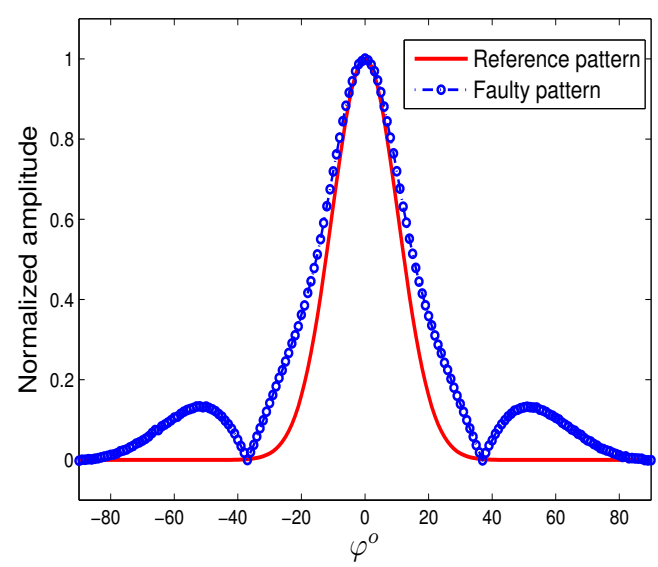

Fig. 2. The reference radiation pattern of the fault-free array (solid curve) and the radiation pattern of the monitored array with completely failing elements at positions -3 and 3 (circle-dash curve), (Case A, first example).

for this case, which is plotted in Figure 3, clearly shows the the violation of the confidence limits and thus the ability of the GLR test to detect this type (on-off) faults.

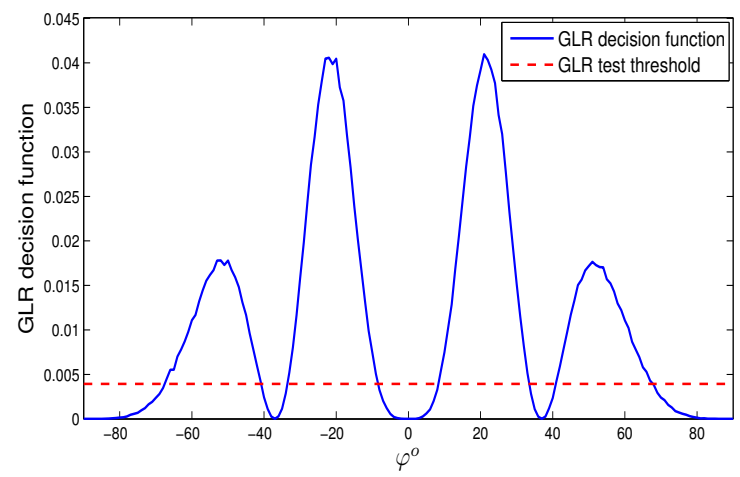

Fig. 3. The evolution of the GLR decision function in the presence of two complete faults at positions -3 and 3 (Case A, first example).

In the second example, an array with two failing elements at positions of -2 and 2 is considered (see Figure 4). Figure 5 shows the radiation patters of the fault-free and faulty arrays, and Figure 9 shows the decision function for this example. As in the first example, Figure 9 shows that the ability of the GLR test to successfully detect these faults. Note, however, that the deviation between the reference and faulty patterns is a function of the position of the faulty elements in the array. 


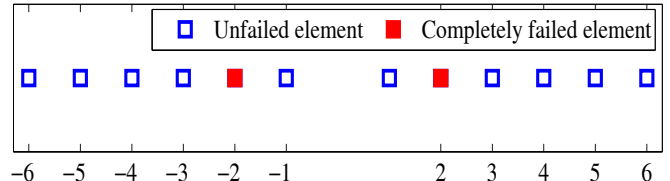

Fig. 4. Example of a linear antenna array with two completely failing elements at positions -2 and 2 (Case A, second example).

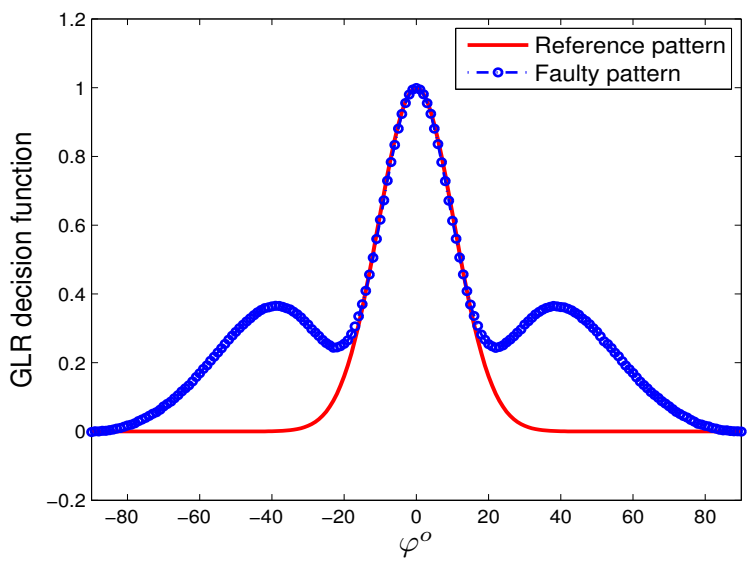

Fig. 5. The reference radiation pattern of the fault-free array (solid curve) and the radiation pattern of the monitored array with completely failing elements at positions -2 and 2 (circle-dash curve), (Case A, second example).

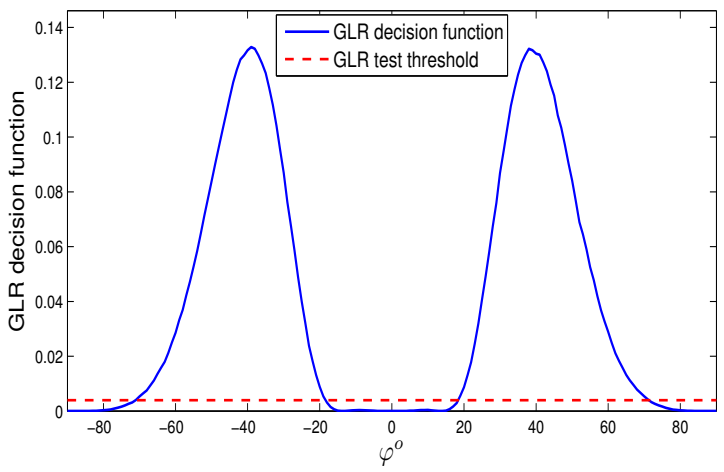

Fig. 6. The evolution of the GLR decision function in the presence of two complete faults at positions -2 and 2 (Case A, second example).

2) Case B: detection of partial failures: The second case is aimed to assess the ability of the GLR test to detect partial failures, i.e., some elements are not completely failing. The partially failing elements are positions -3 and 3 in the linear array (see Figure 7). To simulate the performance of the partially falling array, the excitations of the array are modified to represent the assumed grade of failure. Here, it is assumed that the excitations of the partially failing elements are half of their normal values. Figure 8 shows the radiation patterns of the fault-free array (solid curve) and the faulty array (circledash curve). Again, the false alarm probability is chosen to be $5 \%$ for the GLR test, and the computed decision function is plotted in Figure 9, which shows that the GLR fault detection scheme is capable of detecting this type of partial faults.

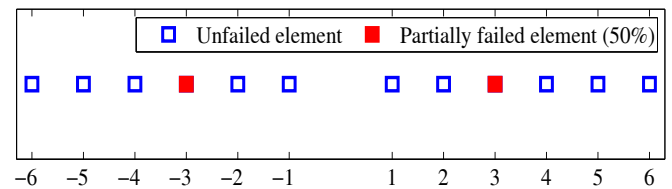

Fig. 7. Example of a linear antenna array with two elements partially failing at positions -3 and 3 (Case B).

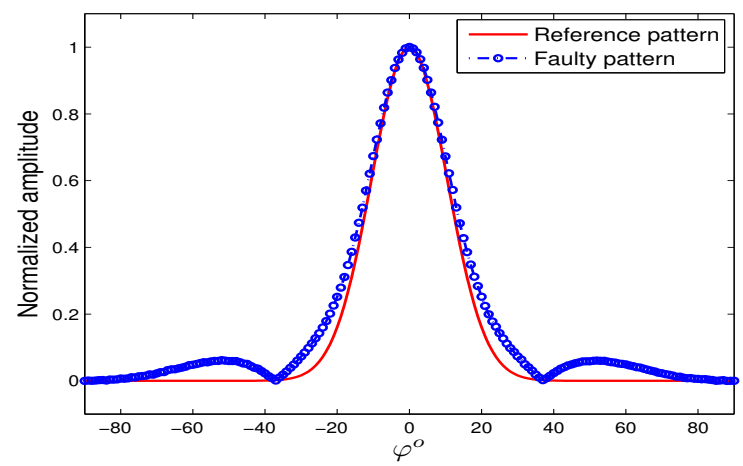

Fig. 8. The reference radiation pattern of the fault-free array (solid curve) and the radiation pattern of the monitored array with partially failing elements at positions -3 and 3 (circle-dash curve), (Case B).

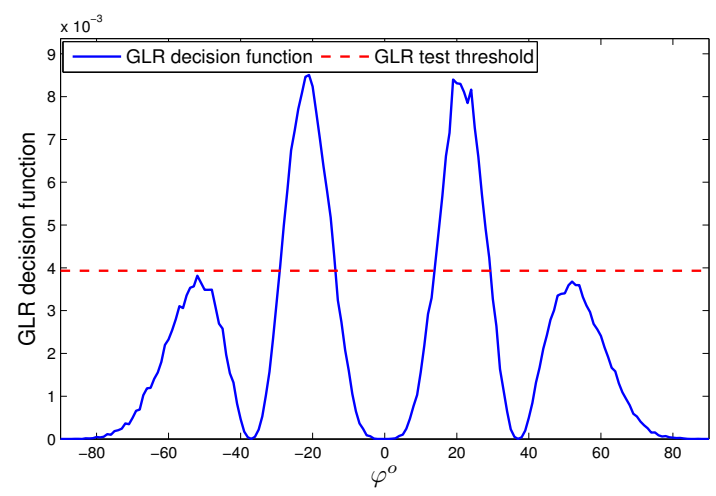

Fig. 9. The evolution of the GLR decision function in the presence of two element partially failing at positions -3 and 3 (Case B).

Note that, in the case of failure(s), the radiation pattern can be restored with a minimal loss of quality (without replacing the defective elements) by re-optimizing the excitations of the antenna elements that did not fail. Several compensation techniques have been developed in the literature, which include machine learning optimization [15], conjugate gradient-based algorithm [16], genetic algorithm [17], simulated annealing technique [18], and many others.

To assess the detection performance of GLR detector, the good detection ratio, which is defined as the percentage of good detection on a certain number of realizations was computed for different value of failure magnitude. To make 
statistically valid conclusions about the performances of this detector, a Monte Carlo simulation using 5,000 realizations is performed for each value of failure magnitude taken between $2 \%$ and $60 \%$. The false alarm probability is chosen to be $5 \%$ for the GLR test. The average of the 5000 of good detection rate as function of failure magnitude is shown in Figure 10.

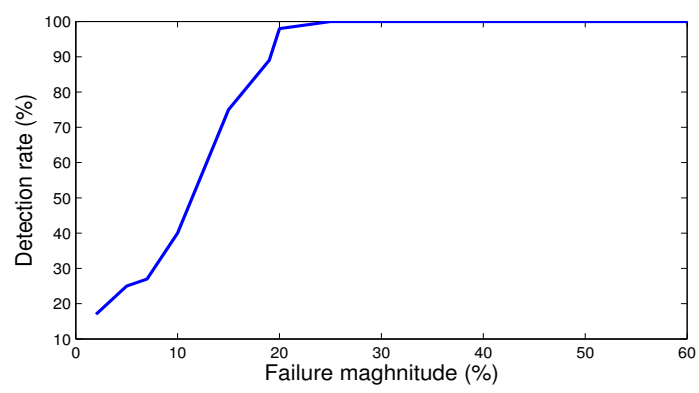

Fig. 10. GLR detector good detection ratio.

3) Case $C$ : detection of interfering signals: In this case study, the ability of GLR test will be assessed through its utilization to detect noise interfering in one or more direction of the radiation pattern of a linear antenna array. Towards this end, two examples are presented. In the first example, the radiation pattern of the monitored linear array is corrupted using random Gaussian noise having a standard deviation of 0.07 in the direction $\varphi=\left[50^{\circ} 70^{\circ}\right]$. The false alarm probability is chosen again to be $5 \%$, and Figure 11, which plots the value of the GLR decision function, shows that this fault is detected by exceeding the threshold value without any false alarms.

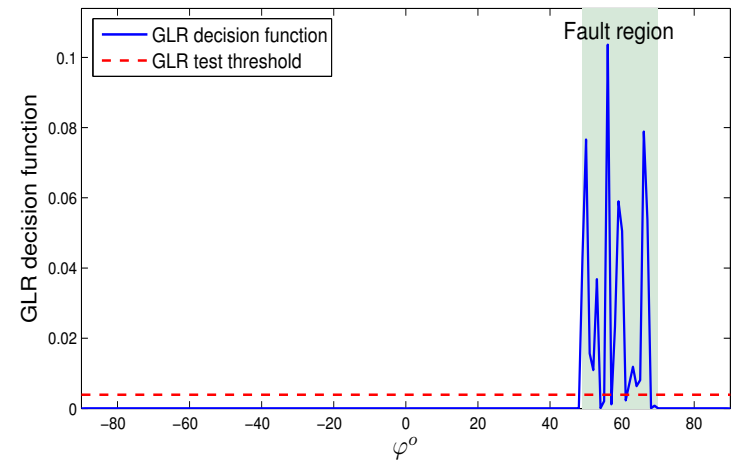

Fig. 11. The evolution of the GLR decision function in the presence of an interfering signal in the direction $\varphi=\left[50^{\circ} 70^{\circ}\right]$, (Case C, first example).

In the second example, the diagnosis of the same array is performed using interfering signals in the directions $\varphi=$ $\left[\begin{array}{ll}-70^{\circ}-50^{\circ}\end{array}\right] \cup\left[\begin{array}{ll}50^{\circ} & 70^{\circ}\end{array}\right]$. The simulation of the radiation pattern representing these faults is performed by introducing two different levels of noise: noise having a standard deviation of 0.05 in the direction $\varphi=\left[-70^{\circ}-50^{\circ}\right]$ and noise having a standard deviation of 0.1 in the direction $\varphi=\left[50^{\circ} 70^{\circ}\right]$. Again, the false alarm probability is chosen to be $5 \%$, and Figure 12, which plots the value of the GLR decision function, shows that this fault is detected by exceeding the threshold value without any false alarms.

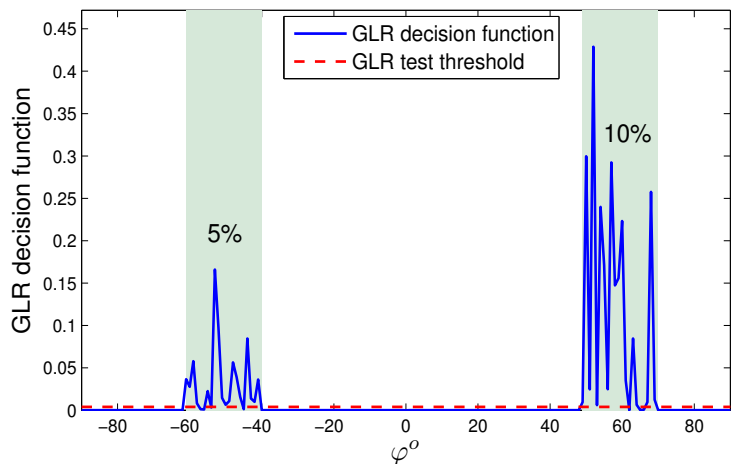

Fig. 12. The evolution of the GLR decision function in the presence of an interfering signal in the directions $\varphi=\left[\begin{array}{ll}-70^{\circ}-50^{\circ} & \cup\left[50^{\circ} 70^{\circ}\right.\end{array}\right]$, (Case $\mathrm{C}$, second example).

The results of these examples show that the GLR hypothesis testing based fault detection method is not only an effective method for detecting the presence of interfering signals, but also it can be used to determine the angle of the interfering signal from which the location of the interference source can be deduced. When such faults are encountered, the excitation of array elements can be re-optimized to produce a pattern with minimal loss of quality with respect to the desired one. To guarantee a reliable performance of the array, noise signals intervening in one or more direction of the radiation pattern can be rejected by numerically finding a new set of excitations of the perturbed array that optimizes some objective function.

\section{CONCLUSION}

With increasing demand for reliability and safety in wireless communication systems, more attention has been given recently by researchers to the problem of fault detection in antenna arrays. The presence of faults changes the radiation pattern of the synthesized array and degrades its performance. In this paper, the problem of fault detection in linear antenna array is addressed from a statistical point of view. Specifically, a fault detection scheme based on the GLR test is proposed to achieve this objective. The proposed method detects faults based on change in the radiation pattern from desired one through three case studies involving 1) complete (on-off) faults, 2) partial faults, and 3) interfering signals. The results showed the effectiveness of the GLR test in detecting these types of faults in a linear antenna array. It is worthwhile to note that the developed methodology can also be be extended to monitor the performances of planar antenna arrays.

\section{REFERENCES}

[1] D. Vakula and N. Sarma, "Fault diagnosis of planar antenna arrays using neural networks," Progress In Electromagnetics Research M, vol. 6, pp. 35-46, 2009.

[2] R. Isermann, "Fault-diagnosis systems: An in troduction from fault detection to fault tolerance," Springer, 2006. 
[3] O. Bucci, A. Capozzoli, and G. D'Elia, "Diagnosis of array faults from far-field amplitude-only data," IEEE Transactions on Antennas and Propagation, vol. 48, no. 5, pp. 647-652, 2000.

[4] F. Harrou and M. Nounou, "Monitoring linear antenna arrays using an exponentially weighted moving average-based fault detection scheme," Systems Science \& Control Engineering: An Open Access Journal, vol. 2, no. 1, pp. 433-443, 2014

[5] M. Carlin, G. Oliveri, and A. Massa, "On the robustness to element failures of linear ads-thinned arrays," IEEE Transactions on Antennas and Propagation, vol. 59, no. 12, pp. 4849-4853, 2011.

[6] G. Oliveri, P. Rocca, and A. Massa, "Reliable diagnosis of large linear arrays-A bayesian compressive sensing approach," IEEE Transactions on Antennas and Propagation, 2012.

[7] G. Castaldi, V. Pierro, and I. Pinto, "Efficient faulty element diagnostics of large antenna arrays by discrete mean field neural nets," Progress In Electromagnetics Research, vol. 25, pp. 53-76, 2000.

[8] C. Balanis, Antenna Theory: Analysis and Design. New York:Wiley, 1982.

[9] T. Ferguson, Mathematical statistics : A decision theoretic approach. New York and London: Academic Press, 1967.

[10] E. Lehmann, Testing Statistical Hypotheses. New York: Chapman and Hall, 1996.

[11] Y. Pawitan, In All Likelihood: Statistical modeling and inference using likelihood. Oxford: Oxford University Press, 2001.

[12] F. Harrou, M. Nounou, H. Nounou, and M. Madakyaru, "Statistical fault detection using PCA-based GLR hypothesis testing," Journal of Loss Prevention in the Process Industries, 2012.

[13] B. Bouyeddou, F. Harrou, S. Djennas, and L. Merad, "Synthesis and optimization of microstrip antennas array using minimax method," in $3 \mathrm{rd}$ European Conference on Antennas and Propagation, EuCAP. IEEE, 2009, pp. 1142-1146.

[14] F. Harrou, B. Bouyeddou, A. Tassadit, and D. Ameziane, "Design of linear and planar microstrip antennas array." International Journal of Information Systems \& Telecommunication Engineering, vol. 1, no. 1, 2010.

[15] N. Xu, C. Christodoulou, S. Barbin, and M. Martínez-Ramón, "Detecting failure of antenna array elements using machine learning optimization," in Antennas and Propagation Society International Symposium, 2007 IEEE. IEEE, 2007, pp. 5753-5756.

[16] T. Peters, "A conjugate gradient-based algorithm to minimize the sidelobe level of planar arrays with element failures," IEEE Transactions on Antennas and Propagation, vol. 39, no. 10, pp. 1497-1504, 1991.

[17] B.-K. Yeo and Y. Lu, "Array failure correction with a genetic algorithm," IEEE Transactions on Antennas and Propagation, vol. 47, no. 5, pp. 823-828, 1999.

[18] J. Rodriguez and F. Ares, "Optimization of the performance of arrays with failed elements using the simulated annealing technique," Journal of electromagnetic waves and applications, vol. 12, no. 12, pp. 16251638, 1998. 